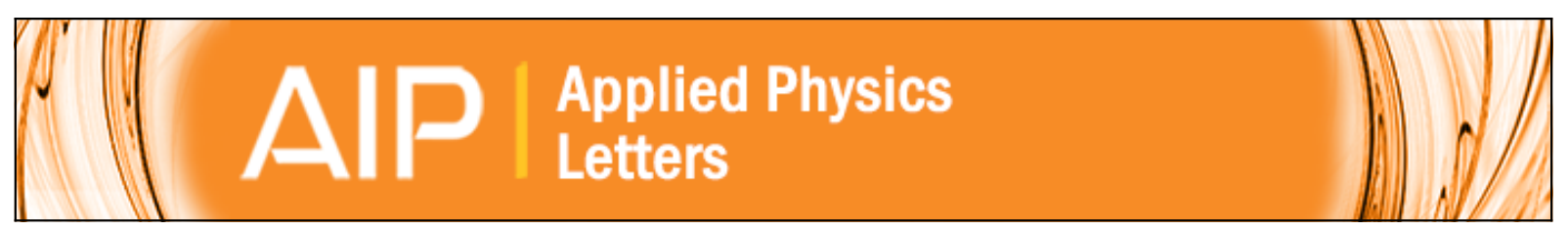

\title{
A nano universal joint made from curved double-walled carbon nanotubes
}

Kun Cai, Haifang Cai, Jiao Shi, and Qing H. Qin

Citation: Applied Physics Letters 106, 241907 (2015); doi: 10.1063/1.4922916

View online: http://dx.doi.org/10.1063/1.4922916

View Table of Contents: http://scitation.aip.org/content/aip/journal/apl/106/24?ver=pdfcov

Published by the AIP Publishing

\section{Articles you may be interested in}

High-speed nano-bearings constructed from double-walled carbon nanotubes: Effect of flexile deformation J. Appl. Phys. 114, 174501 (2013); 10.1063/1.4828871

Molecular dynamics simulation of inertial trapping-induced atomic scale mass transport inside single walled carbon nanotubes

Appl. Phys. Lett. 102, 083108 (2013); 10.1063/1.4793533

Thermal transport in double-wall carbon nanotubes using heat pulse

J. Appl. Phys. 110, 074305 (2011); 10.1063/1.3641970

Coupled oscillations of double-walled carbon nanotubes

J. Appl. Phys. 107, 094310 (2010); 10.1063/1.3359654

Instability analysis of double-walled carbon nanotubes subjected to axial compression

J. Appl. Phys. 104, 036102 (2008); 10.1063/1.2955740

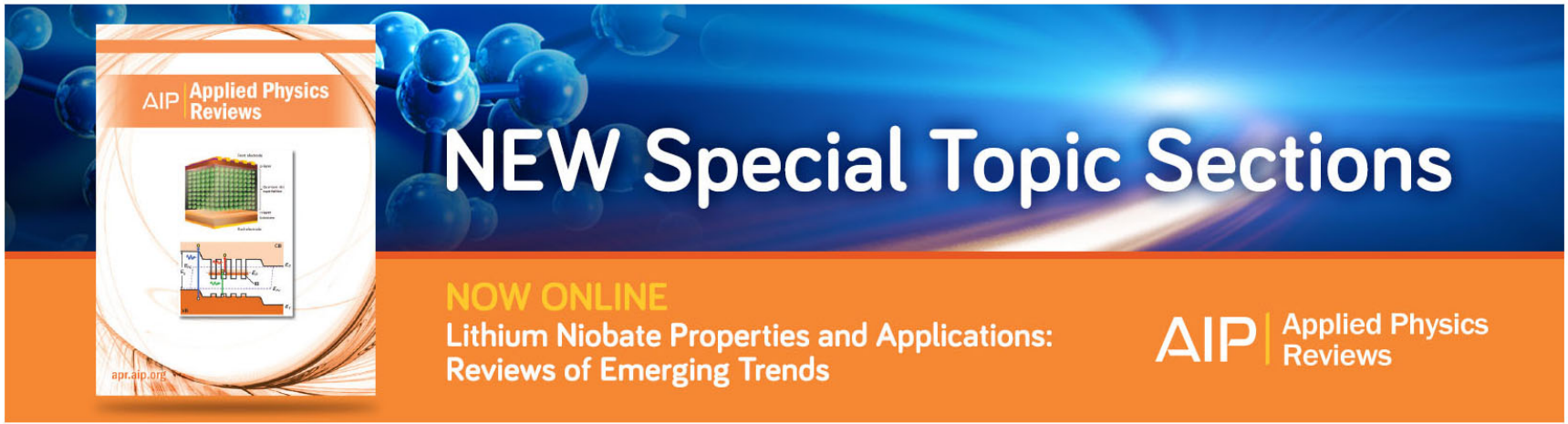




\title{
A nano universal joint made from curved double-walled carbon nanotubes
}

\author{
Kun Cai, ${ }^{1}$ Haifang Cai, ${ }^{1}$ Jiao Shi, ${ }^{1}$ and Qing H. Qin ${ }^{2, \text { a) }}$ \\ ${ }^{1}$ College of Water Resources and Architectural Engineering, Northwest A\&F University, Yangling 712100, \\ China \\ ${ }^{2}$ Research School of Engineering, the Australian National University, Acton, Australian Capital Territory \\ 2601, Australia
}

(Received 20 May 2015; accepted 11 June 2015; published online 19 June 2015)

\begin{abstract}
A nano universal joint is constructed from curved double-wall carbon nanotubes with a short outer tube as stator and a long inner tube as a rotor. When one end of the rotor is driven (by a rotary motor) to rotate, the same rotational speed but with different rotational direction will be induced at the other end of the rotor. This mechanism makes the joint useful for designing a flexible nanodevice with an adjustable output rotational signal. The motion transmission effect of the universal joint is analyzed using a molecular dynamics simulation approach. In particular, the effects of three factors are investigated. The first factor is the curvature of the stator, which produces a different rotational direction of the rotor at the output end. The second is the bonding conditions of carbon atoms on the adjacent tube ends of the motor and the rotor, $s p^{1}$ or $s p^{2}$ atoms, which create different attraction between the motor and the rotor. The third is the rotational speed of the motor, which can be considered as the input signal of the universal joint. It is noted that the rotor's rotational speed is usually the same as that of the motor when the carbon atoms on the adjacent ends of the motor and the rotor are $s p^{1}$ carbon atoms. When they become the new $s p^{2}$ atoms, the rotor experiences a jump in rotational speed from a lower value to that of the motor. The mechanism of drops in potential of the motor is revealed. If the carbon atoms on the adjacent ends are $s p^{2}$ atoms, the rotor rotates more slowly than the motor, whereas the rotational speed is stable when driven by a higher speed motor. (C) 2015 AIP Publishing LLC. [http://dx.doi.org/10.1063/1.4922916]
\end{abstract}

Since they were discovered, carbon nanotubes (CNTs) have attracted much research into their potential. Nowadays, CNTs play a major role in nano-electromechanical systems (NEMS). In particular, the superlubricity ${ }^{1,2}$ between adjacent walls of multiwall CNTs (MWCNTs) promotes their potential application in the design of such nanodevices as gigahertz oscillators, ${ }^{3,4}$ bearings, ${ }^{5,6}$ nanopumps, ${ }^{7,8}$ strain sensors, ${ }^{9-11}$ and motors. ${ }^{12-16}$ Besides superlubricity, CNTs also show strong ductility due to the $s p^{2}-s p^{2}$ covalent bonds within shells. Therefore, the robustness of a nanodevice made from CNTs can be maintained even under extreme environments. Hence, the dynamic responses of such nanodevices have been studied using molecular dynamics (MD) simulation. For example, Zheng and $\mathrm{Jiang}^{3}$ built a gigahertz oscillator using MWCNTs. Qin et al. ${ }^{17}$ studied the mechanical properties of a MWCNT with intramolecular junctions under uniaxial tension. Fennimore et al. ${ }^{18}$ and Bourlon et $a l^{19}$ separately constructed a rotational motor using MWCNTs as the rotation axis. Belikov et al. ${ }^{20}$ studied the screw-like motions of the inner tube in an outer tube with different chiralities. Somada et al. $^{21}$ found the coupling motions of a short-capped CNT within a SWCNT under a varying thermal environment. $\mathrm{Tu}$ and $\mathrm{Hu}^{13}$ and Wang et $a l^{14}$ separately investigated a rotational CNT motor driven by electricity. Cai et $a l .{ }^{22}$ reported a thermally driven rotational motor made from double-wall carbon nanotubes (DWCNTs), with the rotational frequency of the rotor over $100 \mathrm{GHz}$.

\footnotetext{
${ }^{\text {a) }}$ Author to whom correspondence should be addressed. Electronic mail: Qinghua.qin@anu.edu.au
}

The CNTs in the studies mentioned above are intended to be straight in operation. That means that the direction of motion is fixed. In fact, either as a component of a nanodevice or as a nanostructure, the CNT can be curved in operation. Obviously, the dynamic response of a curved CNT may be very different from that of a straight one. ${ }^{23}$ Hence, curved CNTs have attracted increased attention in recent years. For instance, Üstünel et $a l^{24}{ }^{24}$ investigated the vibration behavior of a nanobeam made from a curved CNT. Cai et al. ${ }^{23}$ studied the damped rotational and oscillatory behavior of curved DWCNTs using the MD simulation approach. Mayoof and Hawwa $^{25}$ investigated the chaotic characteristics of a curved SWCNT with a specified configuration of the axis, a sinusoidal curve. Xia and Wang ${ }^{26}$ explored the vibration characteristics of fluid-conveying CNTs with curved axes. The coupled in-plane and out-of-plane motions of a curved CNT was studied by Ouakad and Younis, ${ }^{27}$ who calculated the variation of the nanobeam's natural frequencies and modes with the level of slackness and the direct current (DC) electrostatic load. Ghavanloo et al ${ }^{28}$ conducted in-plane vibration analysis of curved CNTs conveying fluid embedded in a viscoelastic medium. Eichler et al. ${ }^{29}$ developed a technique for detecting the motion of the equilibrium position of the curved nanotube resonator due to symmetry breaking, which was found to actuate the spectral broadening of mechanical resonances. Fakhrabadi et al. ${ }^{30}$ simulated the pull-in charges of SWCNT beams with consideration of the existence of Stone-Wales defects in tubes.

Studies of curved CNTs are similar in such aspects as only SWCNTs being involved, the tube being considered as a beam structure, and the ductility of the SWCNT being 


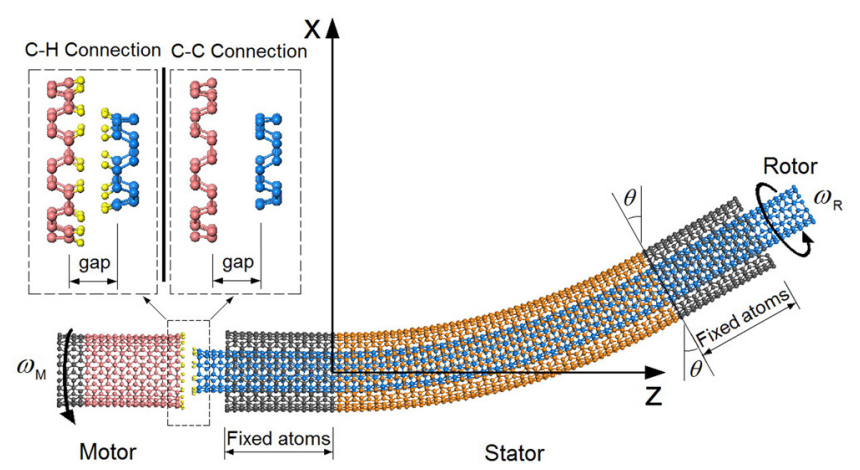

FIG. 1. Configuration of a universal joint made from curved double-walled carbon nanotubes. On the left, the $(9,9)$ motor has 324 carbon atoms with the fixed input rotational speed of $\omega_{\mathrm{M}}$. In the curved joint, the $(5,5)$ rotor (inner tube) with length of $11.473 \mathrm{~nm}$ has 920 atoms, and the $(10,10)$ stator with length of $9.977 \mathrm{~nm}$ has 1600 atoms. The output rotational speed of the rotor is $\omega_{\mathrm{R}}$. At each straight end of the stator, 16 circular layers of (grey) atoms are fixed in simulation. The curved section with orange atoms has isocurvature with the same central angle of $\theta$ within/parallel to the $\mathrm{x}-\mathrm{z}$ plane. The $\mathrm{z}$-axis is along the straight axis; the $\mathrm{x}$-axis is in the $\mathrm{x}-\mathrm{z}$ plane and is perpendicular to the $\mathrm{z}$-axis; the $\mathrm{y}$-axis is normal to the $\mathrm{x}$-z plane. The center of the left 16th layer of the stator is chosen as the origin of the coordinate system, i.e., point $(0,0,0)$. Defining the axial distance between the mass centers of the end circular layers of carbon atoms within the joint as the gap between the motor and rotor, the initial value of the gap is assumed to be $0.34 \mathrm{~nm}$.

implied. Sometimes, however, we may need a device rather than a (nano) structure, such as a device with a universal joint that can transform the original motion into a variety of motions. In the present study, we construct a nano universal joint from curved DWCNTs (Figure 1), in which the outer tube acts as a stator with two fixed ends and the inner tube acts as a rotor. Using the property of the nano universal joint, a more flexible design for a NEMS is possible by setting different curvatures (and lengths) of the DWCNTs to meet the specified needs. A MD approach is employed to find the relations between the input and output rotations of the system.

The rotational transmission model in Fig. 1 is considered in this study. In the numerical simulation, three factors are investigated: the configuration of the connection between motor and rotor, the input rotational frequency $\left(\omega_{M}\right)$ of the motor, and the curvature angle $(\theta)$ of the stator. In the study of the effect of the curvature angle $\theta$ on the dynamic behavior of the nanotube system, three central angles are used in the analysis: $\theta=0^{\circ}, 10^{\circ}$, and $20^{\circ}$. The connection configurations are assumed to be $\mathrm{C}-\mathrm{C}$ and $\mathrm{C}-\mathrm{H}$. With the $\mathrm{C}-\mathrm{C}$ connection, there is no hydrogen atom in the system. Within the $\mathrm{C}$ $\mathrm{H}$ joint, each end carbon atom is covalently bonded with a hydrogen atom. ${ }^{31}$ Two different values of $\omega_{M}$ (input rotational frequency) are considered: 100 and $200 \mathrm{GHz}$. The effects of both the rotational frequency of the rotor, $\omega_{R}$, and the gap in the output are investigated.

In the present simulation, the AIREBO potential ${ }^{32}$ is used to model both covalent bonds between carbon atoms and the long-range van der Waals interactions. Before simulation, $400 \mathrm{ps}$ of relaxation is carried out for the canonical NVT ensemble for $\mathrm{T}=300 \mathrm{~K}$. After relaxation, 5000000 steps of iteration are carried out with the integral time step of $1 \mathrm{fs}$.

In Fig. 2, the dynamic response of the rotor is shown. As the response of the rotor, i.e., $\omega_{\mathrm{R}}$ and gap, can be considered as output signals of the universal joint, the histories of such responses are important in designing a component in a NEMS.

In Fig. 2(a), the rotational frequency of the rotor is the same (in terms of time average) as that of the motor with (a) Rotation of rotor when $\omega_{\mathrm{M}}=100 \mathrm{GHz}$

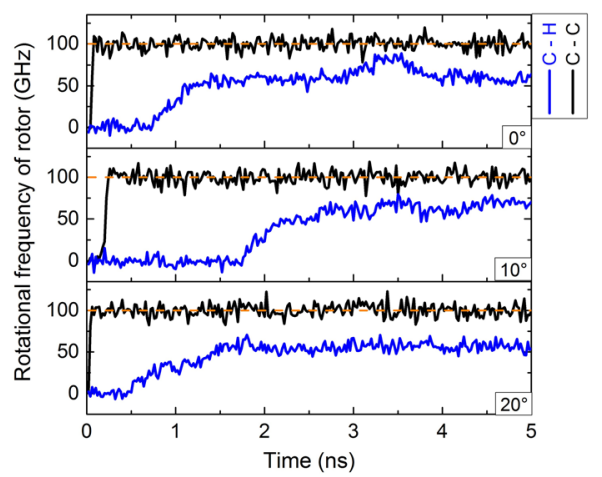

(c) Rotation of rotor when $\omega_{M}=200 \mathrm{GHz}$

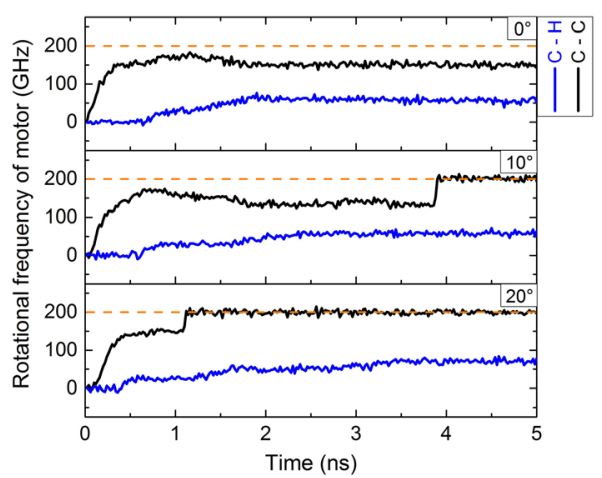

(b) Oscillation of rotor when $\omega_{\mathrm{M}}=100 \mathrm{GHz}$

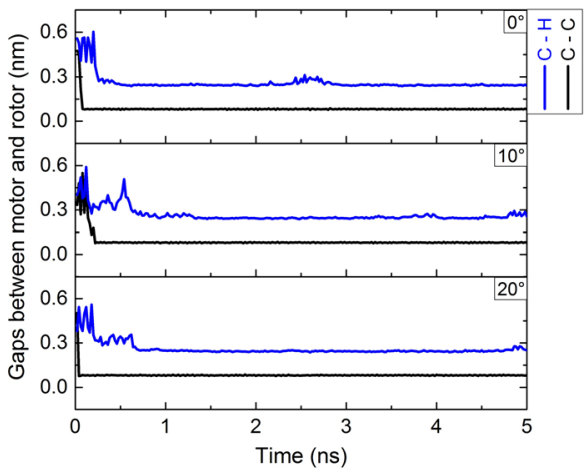

(d) Oscillation of rotor when $\omega_{\mathrm{M}}=200 \mathrm{GHz}$

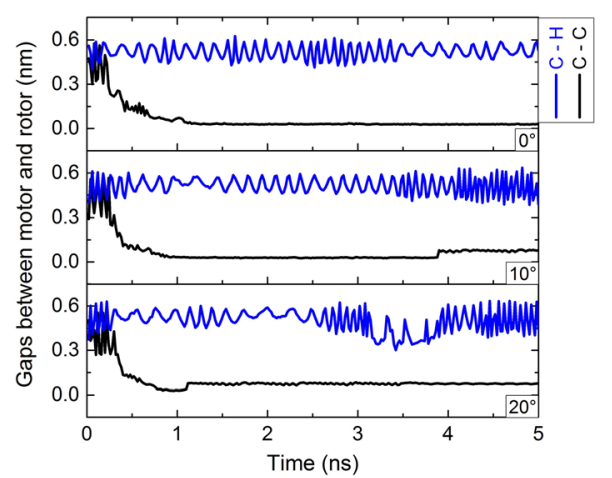

FIG. 2. Output rotational frequency $\left(\omega_{\mathrm{R}}\right)$ and oscillation of the rotor along the curved axis of the universal joint when driven by the rotary motor. The values in the curves are time-averaged within each 20 ps for filtering the heavy fluctuation of data due to the greater thermal vibration of the end carbon atoms in the rotor. (a) Rotation of rotor when $\omega_{\mathrm{M}}=100 \mathrm{GHz}$. (b) Oscillation of rotor when $\omega_{\mathrm{M}}$ $=100 \mathrm{GHz}$. (c) Rotation of rotor when $\omega_{M}=200 \mathrm{GHz}$. (d) Oscillation of rotor when $\omega_{\mathrm{M}}=200 \mathrm{GHz}$. 
(a) Potential energy of $100 \mathrm{GHz}$ motor

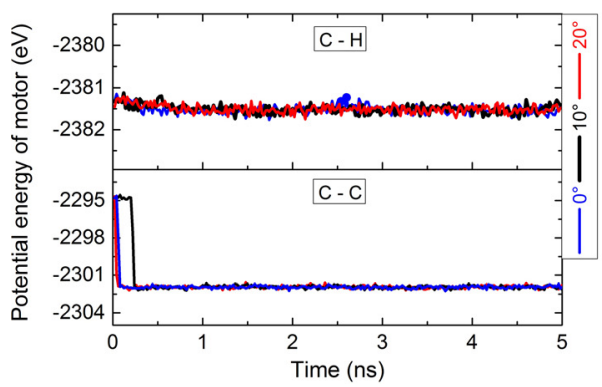

(b) Potential energy of $200 \mathrm{GHz}$ motor

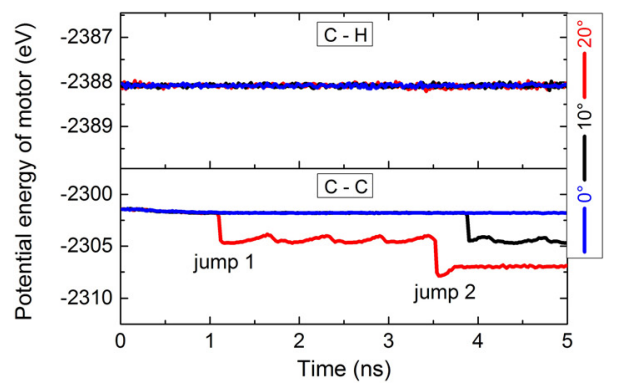

FIG. 3. Potential energy histories of the motor in the system with different connections and the stator with different curvatures.(a) Potential energy of $100 \mathrm{GHz}$ motor. (b) Potential energy of $200 \mathrm{GHz}$ motor.
$100 \mathrm{GHz}$ of rotational frequency when the joint system has a C-C connection (black line). Moreover, the behavior of the rotor is independent of the curvature of the stator. When the system has a $\mathrm{C}-\mathrm{H}$ connection, the rotor rotates asynchronously with the motor (blue line). The rotational speed of the rotor also tends to be stable despite the different curvatures of the stator. The average value of $\omega_{\mathrm{R}}$ in $[3,5] \mathrm{ns}$ is $\sim 75 \mathrm{GHz}$ for the joint with $\theta=0^{\circ}$. When $\theta=10^{\circ}$, the average value of $\omega_{\mathrm{R}}$ is $\sim 72 \mathrm{GHz}$. The rotor has the lowest rotational frequency when the joint has the highest curvature, e.g., $\theta=10^{\circ}$.

We find that the oscillation of the rotor (Fig. 2(b)) is very small after $1000 \mathrm{ps}$ of simulation. For the system with a C-C connection, the stable value of the gap is about $0.082 \mathrm{~nm}$. The spatial distance between the rotor and the motor is very close to the $s p^{2}-s p^{2}$ bond length. This finding implies that the motor and rotor are partly bonded. For the system with a $\mathrm{C}-\mathrm{H}$ connection, the gap stays close to $0.25 \mathrm{~nm}$. It is higher than that in the system with the $\mathrm{C}-\mathrm{C}$ connection, which has stronger attraction between motor and rotor.

In Fig. 2(c), for both $\mathrm{C}-\mathrm{H}$ and $\mathrm{C}-\mathrm{C}$ connections in the universal joint, the rotor's rotational speed differs from that of the $200 \mathrm{GHz}$ motor when the stator is straight, i.e., $\theta=0^{\circ}$. But the rotor's rotational speed in the system with the C-C connection is $150 \mathrm{GHz}$, much higher than that of the rotor in the system with the $\mathrm{C}-\mathrm{H}$ connection, $\sim 56 \mathrm{GHz}$ (the blue line in the upper layer of Fig. 2(c)). In Fig. 2(d), we find that the gap of the $\mathrm{C}-\mathrm{H}$ connection is close to its initial value. At the same time, the gap of the $\mathrm{C}-\mathrm{C}$ connection is close to $0.03 \mathrm{~nm}$. Therefore, we conclude that the different output rotational speed of the rotor is due mainly to the interaction between the $s p^{1}(\mathrm{C}$ in the motor $)-s p^{1}(\mathrm{C}$ in the rotor) being stronger than that in the $\mathrm{C}-\mathrm{H}$ connection (having $s p^{2}-s p^{2}$ carbon atoms).

When the curvature of the stator is not zero and the system has a $\mathrm{C}-\mathrm{C}$ connection, e.g., $\theta=10^{\circ}$ or $20^{\circ}$, the rotational frequency of the rotor evinces a jump during simulation. For example, when $\theta=10^{\circ}$, the jump occurs close to the 3860th ps, after which the rotor rotates synchronously with the motor. From Fig. 2(d), we find that the gap also evinces a jump. When $\theta=20^{\circ}$, the jump occurs close to $1110 \mathrm{ps}$, earlier than that when $\theta=10^{\circ}$. Simultaneously, the gap $\left(\theta=20^{\circ}\right.$, black line in Fig. 2(d)) remains stable at $0.072 \mathrm{~nm}$. These details demonstrate that a higher curvature in the stator leads to a higher radial deviation of the rotor when the length of the left fixed end in the stator is not sufficiently.

There is no jump for either $\omega_{\mathrm{R}}$ or gap when the universal joint has a $\mathrm{C}-\mathrm{H}$ connection. Compared with the results in Fig. 2(a) (driven by the $100 \mathrm{GHz}$ motor), the rotation of the rotor in the curved joint with the $\mathrm{C}-\mathrm{H}$ connection is more stable when driven by the $200 \mathrm{GHz}$ motor. The average rotational frequency of the rotor is $\sim 50 \mathrm{GHz}$ for both $\theta=10^{\circ}$ and $20^{\circ}$.

Fig. 3 shows the potential of the motor during simulations. From the upper layers of Figs. 3(a) and 3(b), we find that the potentials are very close to each other when the universal joint has a $\mathrm{C}-\mathrm{H}$ connection but different rotor curvatures. However, different rotational speeds of the motor lead to different potentials. For example, the average value of the potential of the $100 \mathrm{GHz}$ motor is $\sim-2381.6 \mathrm{eV}$, which is $6.5 \mathrm{eV}$ greater than that of the $200 \mathrm{GHz}$ motor.

When the system has a C-C connection, the potential of the motor evinces jumps at particular time points (the lower layers of Figs. 3(a) and 3(b)). It is easily observed that the first potential jump occurs at the same time as that of the jump in rotational speed of the rotor. Therefore, the sudden acceleration of the rotor's velocity must be due to the sudden variation of the interaction between the motor and the rotor. From the lower layer of Fig. 3(a) and Table I, we find that the potential energy of the $100 \mathrm{GHz}$ motor varies from $\sim-2994.7 \mathrm{eV}$ to $\sim-2301.9 \mathrm{eV}$. The potential drops $\sim 7.2 \mathrm{eV}$.

When the rotational speed of the motor is $200 \mathrm{GHz}$, there are two jumps of potential during driving of the $\theta=20^{\circ}$ rotor. The first jump (jump 1) occurs close to the 1110th ps, and the potential drops by $2.89 \mathrm{eV}$. At the same time, the rotation of the rotor becomes synchronous with that of the motor. The second jump occurs close to the 3530th ps, and

TABLE I. Potential jump of the motor in the system with C-C connection (unit: eV).

\begin{tabular}{lcc}
\hline \hline Curvature (deg) & When $\omega_{\mathrm{M}}=100 \mathrm{GHz}$ & When $\omega_{\mathrm{M}}=200 \mathrm{GHz}$ \\
\hline$\theta=0$ & $-2294.70-(-2301.94)=7.24$ close to 40 th $\mathrm{ps}$ & None \\
$\theta=10$ & $-2294.69-(-2301.93)=7.24$ close to 80 th $\mathrm{ps}$ & $-2301.80-(-2304.48)=2.68$ close to 3860th ps \\
$\theta=20$ & $-2294.67-(-2301.92)=7.25$ close to 20th ps & Jump 1: $-2301.52-(-2304.41)=2.89$ close to 1110th ps \\
& & Jump 2: $-2304.39-(-2306.98)=2.59$ close to 3530th ps \\
\hline
\end{tabular}


(a) Configurations near jump 1

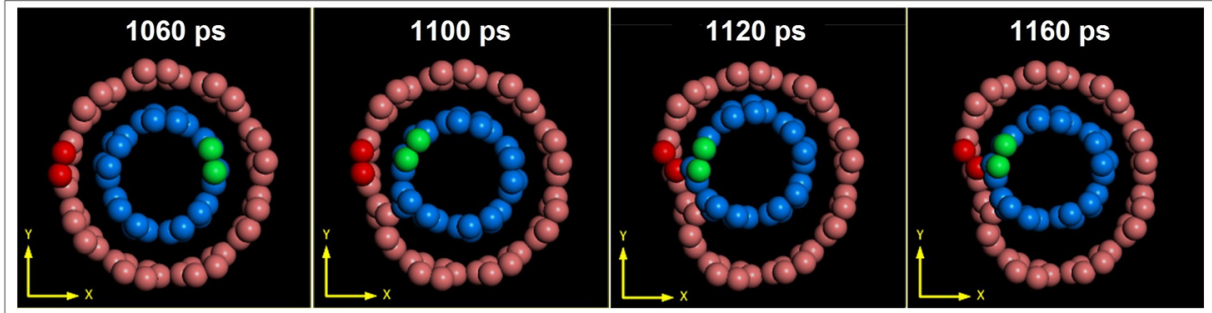

(b) Configurations near jump 2
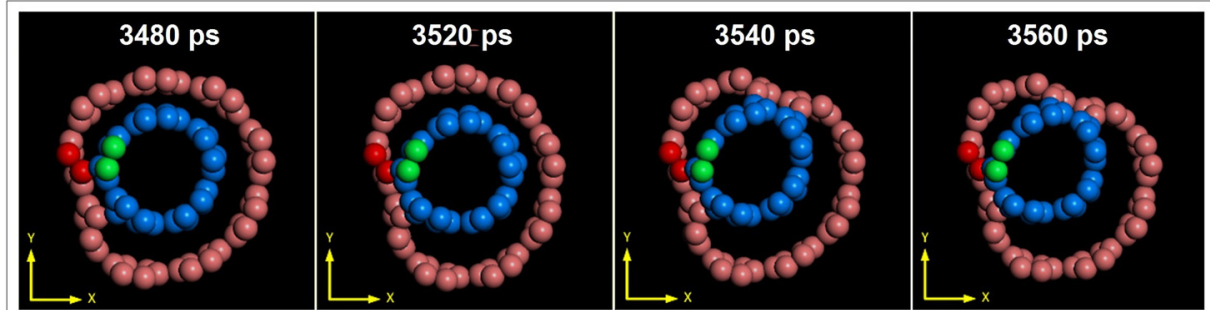

the potential drops by $2.59 \mathrm{eV}$. It is known that the $s p^{1}$ and $s p^{2}$ binding energy of a carbon atom are at -283.5 and $-284.4 \mathrm{eV}$, respectively. ${ }^{33}$ The difference is $\sim 0.9 \mathrm{eV}$. From the results, we find that the drops in potential for the two jumps are about 3 times the difference between the binding energies of $s p^{1}$ and $s p^{2}$ of the carbon atom. In Fig. 4(a), the configurations of the $\mathrm{C}-\mathrm{C}$ connection are shown, implying that "jump 1" occurs during $[1100,1120]$ ps. After the 1120 th ps, there are always 3 carbon atoms near the red atoms (on the motor) very close to the rotor. This finding implies that at least one $s p^{1}$ carbon atom on the motor has become a $s p^{2}$ atom. Simultaneously, near the new $s p^{2}$ atoms, some other atoms are also closer to the rotor. The two phenomena create the decrease in potential of the motor. Similarly, some carbon atoms on the motor become $s p^{2}$ atoms when "jump 2" occurs during [3520, 3540] ps. Therefore, we conclude that the new covalent bonds between the motor and the rotor lead to the rotor rotating synchronously with the motor that has a fixed rotational speed.

For the system with C-C connection and the $\theta=20^{\circ}$ rotor driven by the $200 \mathrm{GHz}$ motor, the first drop in potential is $2.89 \mathrm{eV}$ (Table I), which is more than 3 times the difference between the binding energies of $s p^{1}$ and $s p^{2}$ of the carbon atom. But the second drop in potential, i.e., $2.59 \mathrm{eV}$, is less than 3 times the difference. The reason can be found from Fig. 4. For example, in Fig. 4(a), at the 1120th ps, the deformation of the motor is greater than that of the rotor, because of the difference between the in-shell bending stiffness of motor and rotor. For the first deformation of the motor, there is only one location with heavy deformation at the end. After the first deformation, the in-shell bending stiffness of the motor is greater than that before deformation. Therefore, the second deformation occurs with smaller drop in potential.

Financial support from the National Natural-ScienceFoundation of China (Grant Nos. 50908190 and 11372100) is gratefully acknowledged.
FIG. 4. Configurations of the C-C connection between the $200 \mathrm{GHz}$ motor (grey atoms) and the $\theta=20^{\circ}$ rotor (blue atoms) near jumps in potential. The two red atoms mark the position of the motor in the coordinate system (x-y). (a) Configurations near jump 1. (b) Configurations near jump 2.
${ }^{1}$ J. Cumings and A. Zettl, Science 289, 602 (2000).

${ }^{2}$ R. Zhang, Z. Ning, Y. Zhang, Q. Zheng, Q. Chen, H. Xie, Q. Zhang, W. Qian, and F. Wei, Nat. Nanotechnol. 8, 912 (2013).

${ }^{3}$ Q. Zheng and Q. Jiang, Phys. Rev. Lett. 88, 045503 (2002).

${ }^{4}$ K. Cai, H. Yin, Q. H. Qin, and Y. Li, Nano Lett. 14, 2558 (2014).

${ }^{5}$ S. Zhang, W. K. Liu, and R. S. Ruoff, Nano Lett. 4, 293 (2004).

${ }^{6}$ C. Zhu, W. Guo, and T. Yu, Nanotechnology 19, 465703 (2008).

${ }^{7}$ S. Joseph and N. Aluru, Phys. Rev. Lett. 101, 064502 (2008).

${ }^{8}$ A. Lohrasebi and Y. Jamali, J. Mol. Graphics Modell. 29, 1025 (2011).

${ }^{9}$ W. Qiu, Q. Li, Z. Lei, Q. H. Qin, W. Deng, and Y. Kang, Carbon 53, 161 (2013).

${ }^{10}$ W. Qiu, Y. Kang, Z. Lei, Q. H. Qin, and Q. Li, Chin. Phys. Lett. 26, 080701 (2009).

${ }^{11}$ W. Qiu, Y. L. Kang, Z. K. Lei, Q. H. Qin, Q. Li, and Q. Wang, J. Raman Spectrosc. 41, 1216 (2010).

${ }^{12}$ J. W. Kang and H. J. Hwang, Nanotechnology 15, 1633 (2004).

${ }^{13}$ Z. Tu and X. Hu, Phys. Rev. B 72, 033404 (2005).

${ }^{14}$ B. Wang, L. Vuković, and P. Král, Phys. Rev. Lett. 101, 186808 (2008).

${ }^{15}$ K. Cai, Y. Li, H. Yin, and Q. H. Qin, Mech. Ind. 16, 110 (2015).

${ }^{16}$ K. Cai, J. Yu, H. Yin, and Q. H. Qin, Nanotechnology 26, 095702 (2015).

${ }^{17}$ Z. Qin, Q. H. Qin, and X. Q. Feng, Phys. Lett. A 372, 6661 (2008).

${ }^{18}$ A. M. Fennimore, T. D. Yuzvinsky, W. Q. Han, M. S. Fuhrer, J. Cumings, and A. Zettl, Nature 424, 408 (2003).

${ }^{19}$ B. Bourlon, D. C. Glattli, C. Miko, L. Forró, and A. Bachtold, Nano Lett. 4, 709 (2004).

${ }^{20}$ A. Belikov, Y. E. Lozovik, A. Nikolaev, and A. Popov, Chem. Phys. Lett. 385, 72 (2004).

${ }^{21}$ H. Somada, K. Hirahara, S. Akita, and Y. Nakayama, Nano Lett. 9, 62 (2009).

${ }^{22}$ K. Cai, Y. Li, Q. H. Qin, and H. Yin, Nanotechnology 25, 505701 (2014).

${ }^{23}$ K. Cai, H. Cai, H. Yin, and Q. H. Qin, RSC Adv. 5, 29908 (2015).

${ }^{24}$ H. Üstünel, D. Roundy, and T. Arias, Nano Lett. 5, 523 (2005).

${ }^{25}$ F. N. Mayoof and M. A. Hawwa, Chaos, Solitons Fractals 42, 1860 (2009).

${ }^{26}$ W. Xia and L. Wang, Comput. Mater. Sci. 49, 99 (2010).

${ }^{27}$ H. M. Ouakad and M. I. Younis, J. Sound Vib. 330, 3182 (2011).

${ }^{28}$ E. Ghavanloo, M. Rafiei, and F. Daneshmand, Phys. Lett. A 375, 1994 (2011).

${ }^{29}$ A. Eichler, J. Moser, M. Dykman, and A. Bachtold, Nat. Commun. 4, 2843 (2013).

${ }^{30}$ M. M. S. Fakhrabadi, P. K. Khorasani, A. Rastgoo, and M. T. Ahmadian, Solid State Commun. 157, 38 (2013).

${ }^{31}$ A. M. Popov, I. V. Lebedeva, A. A. Knizhnik, Y. E. Lozovik, and B. V. Potapkin, J. Chem. Phys. 138, 024703 (2013).

${ }^{32}$ S. J. Stuart, A. B. Tutein, and J. A. Harrison, J. Chem. Phys. 112, 6472 (2000).

${ }^{33}$ A. Hu, M. Rybachuk, Q. B. Lu, and W. Duley, Appl. Phys. Lett. 91, 131906 (2007). 